\title{
Management and Treatment for Cerebral Palsy in Children
}

\author{
Padmakar $\mathbf{S}^{1 *}$, K Sujan Kumar', S Parveen ${ }^{2}$ \\ ${ }^{1}$ Pharm-D, P. Rami Reddy Memorial College of Pharmacy [PRRMCP], Kadapa, Andhra Pradesh, INDIA. \\ ${ }^{2}$ Department of Pharmacy Assitant Professor, P. Rami Reddy Memorial College of Pharmacy [PRRMCP], Kadapa, \\ Andhra Pradesh, INDIA.
}

\begin{abstract}
'Cerebral' - refers to the brain. 'Palsy' - can mean weakness or paralysis or lack of muscle control. Therefore, cerebral palsy is a disorder of muscle control which results from some damage to part of the brain. Cerebral palsy (CP) is a group of permanent disorders of the development of movement and posture, causing activity limitation, that are attributed to non-progressive disturbances that occurred in the developing foetal or infant brain. The motor disorders of cerebral palsy are often accompanied by disturbances of sensation, perception, cognition, communication, and behaviour, by epilepsy, and by secondary musculoskeletal problems. Approximately $80 \%$ to $90 \%$ of children with cerebral palsy have spastic cerebral palsy. The diagnosis of spasticity in children with CP requires a complete physical examination, with ancillary testing as needed. The aim of treatment is to encourage the child to learn to be as independent as possible. Some children who have mild cerebral palsy will not have any problems in achieving independence. For others, it will be a slow process. In some with severe difficulties, considerable assistance from others will always be needed. Specific treatment varies by individual and changes as needed if new issues develop. In general, treatment focuses on ways to maintain or improve a person's quality of life and overall health. The goal of management of cerebral palsy is not to cure or to achieve normalcy but to increase functionality, improve capabilities, and sustain health in terms of locomotion, cognitive development, social interaction, and independence.
\end{abstract}

Key words: Cerebral palsy, Management, Treatment, Children.

\section{INTRODUCTION}

\section{DEFINITION}

Cerebral Palsy is a group of permanent, but not unchanging, disorders of movement and/or posture and of motor function, which are due to a non-progressive interference, lesion, or abnormality of the developing/ immature brain. ${ }^{1}$

Cerebral palsy is primarily a disorder of movement and posture. It is defined as an "umbrella term covering a group of nonprogressive, but often changing, motor impairment syndromes secondary to lesions or anomalies of the brain arising in the early stages of its development". ${ }^{2}$

\section{EPIDEMOLOGY}

Unfortunately, it is difficult to access and clarify the prevalence and incidence rate of disabilities in poor-resource settings (Gladstone, 2010). Not only the prevalence of childhood disability is on the rise and Cerebral Palsy is one of the costliest chronic conditions, but also life expectancies are improving, which increases the burden of Cerebral Palsy (Papavasiliou, 2009). For comparison, in the USA, there are approximately $700^{\prime} 000$ children with Cerebral Palsy, 2-5/ 1000 born.

Cerebral Palsy is the most common motor disability in childhood. The aetiology of Cerebral Palsy is very diverse and multifactorial. The causes are congenital, genetic, inflammatory, infectious, anoxic, traumatic and metabolic. Population-based studies from around the world report prevalence estimates of Cerebral Palsy ranging from 1.5 to more than 4 per 1,000 live births or
DOI: 10.5530/ijopp.11.2.23

Address for correspondence:

Padmakar S,

Pharm.d intership,P. Rami Reddy Memorial College of Pharmacy [PRRMCP], Kadapa, Andhra Pradesh, INDIA.

Phone no: 9603656446

Email Id: spadmakar717@gmail. com

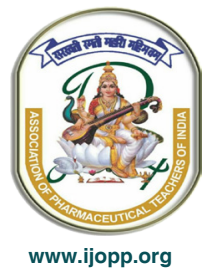


children of a defined age range. Most of the children identified with Cerebral Palsy have Spastic Cerebral Palsy (77, 4\%). Over half of the children identified with Cerebral Palsy $(58,2 \%)$ can walk independently, 11, 3\% walks using a handheld mobility device and $30,6 \%$ has limited or no walking ability. Many children with Cerebral Palsy also do have at least one co-occurring condition (e.g. $41 \%$ Epilepsy). ${ }^{3}$

\section{TYPES OF CEREBRAL PALSY}

There are three types of cerebral palsy that can be distinguished by their symptoms and management approaches. The man types of $\mathrm{CP}$ are Spastic, Ataxic and Athetoid cerebral palsy. ${ }^{4}$

A. Spastic Cerebral Palsy: This is the most common type of CP. Spastic CP is characterized by unique muscle tightness, patients have muscle spasticity as the main impairment characteristic. ${ }^{5}$ This type of $\mathrm{CP}$ occurs in at least $70 \%$ of all CP cases in the world. In cases of spastic CP, the disorder is more easily manageable as compared to other types since treatment through medication can be pursued in several neurological and orthopaedic approaches. The spasticity of muscles leads to other muscle stress symptoms that may include tendinitis and arthritis in individuals who are 20-30 years old. This type of CP can be managed using occupational and physical therapy where strengthening, stretching, exercise and other physical activities are used to manage the disorder on a daily basis. The disorder can also be managed using medications that eliminate spasticity by killing the very nerves that cause the disorder. ${ }^{5}$

B Ataxic Cerebral Palsy: This type of $\mathrm{CP}$ is less common as compared to spasticity, it may occur in $6-10 \%$ of all cases of CP. Ataxic CP is characterized by "ataxia-type" symptoms that inflict some cerebellum damage. The child may exhibit symptoms of unsteady posture. One may also shake while attempting to hold objects with the hand. Such symptoms are part of the motor degraded motor skills experienced by the child. One may have difficulties in their control of motor skills, which include typing, writing and holding small objects. The child may also show some disorientation and poor control while walking. Visual and auditory processing may also be affected in ataxic CP.

C Athetoid Cerebral Palsy: This is also called Dyskinetic $\mathrm{CP}$; it occurs in at least $10 \%$ of all CP cases. As compared to spasticity, the occurrence of this type of CP is relatively low. Patients with this type of disorder may have challenges in maintaining steady positioning. Steady sitting and walking is quite problematic; individuals may show some unintended

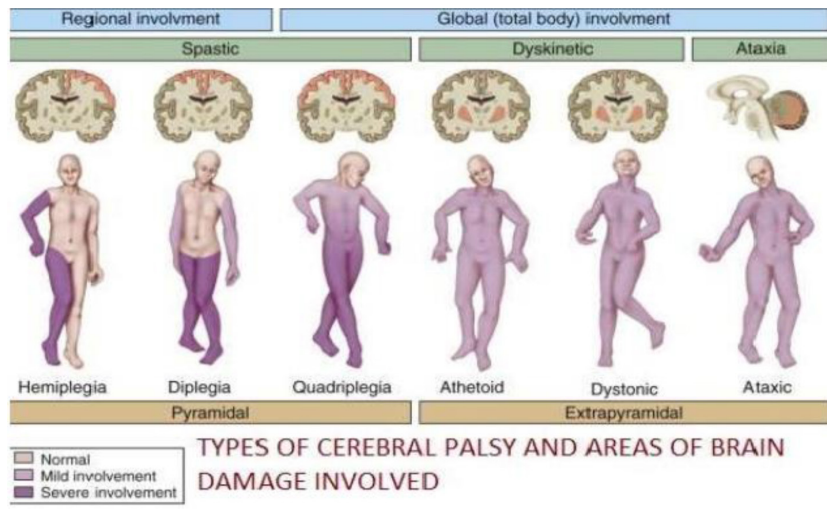

Figure 1: TYPES OF CEREBRAL PLASY

motions. In addition, patients may lose their ability to hold objects especially small objects that require some fine or advanced motor control. Such patients may not be able to hold small objects such as pens, coins and other small objects. ${ }^{5}$

\section{CLINICAL RISK FACTORS FOR CP DURING PREGNANCY}

There is increasing scientific evidence that $\mathrm{CP}$ is usually associated with longstanding intrauterine pathology like genetic mutations and probable environmental triggers such as bacterial and viral intrauterine infection, intrauterine growth restriction (IUGR), antepartum hemorrhage, tight nuchal cord, and threatened miscarriage. ${ }^{6} \mathrm{It}$ can be difficult to pinpoint adverse pregnancy factors in retrospect, many years after birth, that individually or together might have triggered the pathways to the neuropathology.

\section{Preterm delivery}

Preterm delivery is a major risk factor for $\mathrm{CP}$ and is seen in approximately $35 \%$ of all cases, and the risk increases the lower the viable gestational age. ${ }^{6,7}$ The risk of subsequent $\mathrm{CP}<33$ weeks' gestation is 30 times higher than among those born at term and is approximately $70 / 1000$ deliveries. 7 The prevalence of $\mathrm{CP}$ is highest in children born $<28$ weeks' gestational age $(111.8 / 1000$ neonatal survivors; 82.25/1000 live births) and declines with increasing gestational age, being 43.15/1000 live births between 28-31 weeks, $6.75 / 1000$ between 32-36 weeks, and 1.35/1000 for those born $>36$ weeks. ${ }^{8}$ The mechanisms and pathways to the neuropathology of CP may differ from term babies, although associated risk factors such as infection, genetic variations, and growth restriction are likely to contribute.

\section{Coexisting congenital anomalies}

The prevalence of congenital anomalies in children with $\mathrm{CP}$ is much higher than in the general population 
and most are cerebral, such as schizencephaly and hydrocephaly. ${ }^{9}$ Non-cerebral malformations are also increased, such as cardiac, musculoskeletal, and urinary. ${ }^{10}$ In a case-control study of 494 singleton infants with CP born $>35$ weeks' gestation included on the Western Australian Register of Developmental Anomalies and 508 matched controls, birth defects (42.3\%) and fetal growth restriction $(16.5 \%)$ were more strongly associated with CP than potentially asphyxial birth events $(8.5 \%)$ and inflammation (4.8\%). ${ }^{11}$ Birth defects had the largest association with $\mathrm{CP}$ in that study in both term and preterm babies. Growth restricted babies with birth defects were at special risk of CP. The strong association with congenital abnormalities suggests possible genetic factors although congenital infections, nutritional disorders, and teratogenic influences all contribute to maldevelopment.

\section{INTRAUTERINE INFECTION}

There are many probable antenatal causes of whitematter damage and risk factors for CP. Some of these causes include damage acquired following perinatal infection (i.e., maternal infection that affects the foetus and its brain during pregnancy and/or labour or in the neonatal period). ${ }^{12}$ Viral or bacterial infections may be relatively silent during pregnancy and not recognized clinically at the time and the placenta is often discarded without histological examination for inflammatory pathology. Maternal reports of fever or infection during pregnancy are significantly associated with an increased risk of CP in our recent large Australian case-control study. ${ }^{13}$ Evidence of intrauterine infection, evidenced by histological chorioamnionitis in the placenta and membranes or intrapartum pyrexia, is associated with a 4-fold increase in CP (odds ratio 3.8; 95\% confidence interval, $1.5 \mathrm{e} 10.1)$ in term infants. ${ }^{14}$

\section{Intrauterine growth restriction}

IUGR is associated with up to a 10 to 30 fold increase in the risk of $\mathrm{CP}$ at term. ${ }^{15}$ In particular, spastic CP increases with the degree of fatal growth restriction. A growth-restricted foetus may show signs of possible fatal compromise during labour. This can reflect reduced capacity/reserves to withstand the normal stresses of labour, established neurological and ongoing fatal compromise, or both. It is not possible to distinguish between these timings.

\section{Multiple pregnancy}

Multiple pregnancy increases CP risk 2-fold in each twin. In vitro fertilization twins each have $>4$-fold risk $(9.5 / 1000)$, giving another reason to encourage single embryo transfer in fertility programs. ${ }^{16}$

\section{Viral infection in pregnancy}

Studies using polymerase chain reaction techniques on neonatal blood spots from CP cases and controls show increased CP risk after both Cytomegalovirus and Epstein-Barr virus infections during pregnancy. ${ }^{17}$ Epidemiological studies do not associate upper respiratory infections during pregnancy with $\mathrm{CP}$, but some studies have associated increased risk with bacterial urinary tract infections. ${ }^{12,18}$

\section{Genetic causes of CP}

Genetic causes have long been suspected because of the link with congenital malformations, and increased risk in consanguineous families and monozygotic twins. 43 Although initially candidate gene association studies suggested that several genes may be linked to $\mathrm{CP}$, the power of these studies was low and multiple comparisons weakened their validity. ${ }^{19,20}$

\section{DIAGNOSIS}

Observation of slow motor development, abnormal muscle tone, and unusual posture are common initial clues to the diagnosis of cerebral palsy. Assessment of persistent infantile reflexes is important. In infants who do not have cerebral palsy, the Moro reflex is rarely present after six months of age, and hand preference rarely develops earlier than 12 months of age. Hand preference may occur before 12 months of age if spastic hemiplegia is present. $^{21}$ Progressive hereditary neurologic or metabolic disorders must be eliminated as the cause of observed abnormalities. The testing strategy is based on the clinical picture, pattern of development of symptoms, family history, and other factors influencing the probability of specific diagnoses. Targeted laboratory tests and cerebral imaging using computed tomography, magnetic resonance imaging, and ultrasound are useful physical diagnostic tools. Surveillance for associated disabilities such as hearing and vision impairment, seizures, perception problems with touch or pain, and cognitive dysfunction can help complete the clinical assessment and determine the diagnosis. ${ }^{22}$

\section{MANAGEMENT}

The goal of management of cerebral palsy is not to cure or to achieve normalcy but to increase functionality, improve capabilities, and sustain health in terms of locomotion, cognitive development, social interaction, and independence. The best clinical outcomes result from early, intensive management. Optimal treatment in children requires a team approach. ${ }^{23} \mathrm{~A}$ modern team approach focuses on total patient development, not just on improvement of a single symptom. Treatment 
programs encompass physical and behavioural therapy, pharmacologic and surgical treatments, mechanical aids, and management of associated medical conditions. In physical, occupational, speech, and behavioural therapies, the goals include enhancing patient and caregiver interactions while providing family support. ${ }^{23}$

Management of spasticity is a major challenge to treatment team. Various forms of therapy are available to people living with cerebral palsy as well as caregivers and parents caring for someone with this disability. They can all be useful at all stages of this disability and are vital in a CP person's ability to function and live more effectively. ${ }^{24}$

\section{Oral Medications}

Oral medications are a systemic, rather than focal, treatment for spasticity in children with cerebral palsy. Oral medications commonly used in children are baclofen, diazepam, clonazepam, dantrolene and tizanidine..$^{25}$

\section{Intrathecal Baclofen}

Intrathecal baclofen (ITB) was approved for the treatment of spasticity of cerebral origin in 1996. ITB is a surgically implanted system used to control spasticity by infusing baclofen directly into the spinal canal and around the spinal cord. ${ }^{26}$ Baclofen inhibits spasticity by blocking excitatory neurotransmitters in the spinal dorsal horn. ITB maximizes the dose delivered to spinal receptors and minimizes the side effects associated with oral baclofen. ${ }^{27}$

\section{Botulinum Toxin}

Botulinum toxin (BT) injection is now an established first-line treatment for focal spasticity. Botulinum toxin type A produces dose-related weakness of skeletal muscle by impairing the release of acetylcholine at the neuromuscular junction. This partially interrupts muscle contraction making the muscle temporarily weaker. Muscles commonly treated with BT include the gastrocnemius-soleus complex, hamstrings, hip adductors and flexor synergy muscles of the upper extremity. Intramuscular injections can be localized by surface landmarks, electromyography stimulation, and/or ultrasound. Following injection, muscle relaxation is evident within 48 to $72 \mathrm{hr}$ and persists for a period of 3 to 6 months. Botox injection can help improve a child's ability to walk or use hands and allow for a better fitting orthotics by reducing spasticity. Therapists can take advantage of the time when an overly powerful muscle is weakened to work on strengthening the muscle on the opposite side of the joint (antagonist). Sometimes, casting of the involved extremity is done after the injection to increase the stretch of the tight muscle. ${ }^{28-32}$

Indian Journal of Pharmacy Practice, Vol 11, Issue 2, Apr-Jun, 2018

\section{SURGERY}

\section{Orthopedic surgery}

Surgery is mainly undertaken on the lower limb, but occasionally in the upper limb. Some children require surgery for scoliosis. Physiotherapy is an essential part of post-operative management. Gait laboratories are useful in planning the surgical program for children who can walk independently or with sticks or walking frames.

- The hip: soft tissue surgery is often effective for children when the hip problems are detected at an early stage (hence the importance of regular $\mathrm{X}$-rays). Lengthening of the adductor muscles may be all that is required in younger children. However, if the problem progresses, and especially if it is neglected, more extensive surgery to the hip bones is required for a significant number of children. For most children surgery to keep the hips in joint, or to put the hips back in joint, is preferable to leaving the child with a dislocated hip which is frequently painful in later life.

- The knee: lengthening of the hamstrings can help the knee straighten and so improve the walking pattern. Sometimes transferring a muscle from the front to the back of the knee can also help by reducing stiffness around the knee.

- The ankle and foot: This is the commonest area where orthopedic surgery is required. Sometimes children require orthopedic surgery in several different areas (for example, hip, knee and ankle). Frequently this now involves a single hospitalization and is called 'multilevel surgery'. Multilevel surgery is of most benefit to children whowalk independently or with the assistance of crutches.

The best age is usually between 8 and 12 years old although it can occasionally be helpful for older or younger children. ${ }^{33}$

\section{TREATMENT FOR THE ASSOCIATED MEDICAL PROBLEMS}

\section{Epilepsy}

Knowledge of epilepsy has increased substantially in the past few years. There are many types of epilepsy, and medication is often prescribed following a careful diagnosis of the type of seizures and their cause. The most commonly used anticonvulsants are: Carbamazepine, Sodium valproate, Lamotrigine, Phenytoin etc.

\section{Saliva control}

The speech pathologist plays a central role and can provide strategies to improve dribbling problems. When these strategies are not effective, medication is 
occasionally used, particularly in children over the age of six years. These medications are as follows:

- Benzhexol hydrochloride ('Artane') reduces salivary secretions. Occasionally irritability may occur. Blurring of vision, constipation and difficulty with urination are also potential side effects.

- lycopyrrolate ('Robinul') is like benzhexol hydrochloride but seems to produce fewer side effects.

\section{Constipation}

Children with cerebral palsy often have problems with constipation. A high fiber diet and increased fluid intake can help with this problem. This may not be easily achieved in some children with cerebral palsy. Careful use of laxatives is preferable to severe constipation.

\section{Nutrition}

A dietitian can provide useful advice about adequate nutrition. Excessive weight gain can be very disadvantageous for children learning to walk. Under nutrition and failure to make adequate weight gains may be related to feeding difficulties. In a small proportion of children, the use of tube feeding can be helpful. ${ }^{33}$

\section{CONCLUSION}

Cerebral palsy is a disorder of muscle control which results from some damage to part of the brain. Children with cerebral palsy can have problems such as muscle weakness, stiffness, awkwardness, slowness, shakiness, and difficulty with balance which remain throughout the lifetime of a person. The goal of management of cerebral palsy is not to cure or to achieve normalcy but to increase functionality, improve capabilities, and sustain health in terms of locomotion, cognitive development, social interaction, and independence. The management often requires a variety of different approaches including oral medications, botulinum toxin, intrathecal baclofen, occupational and physical therapy and often surgical interventions.

\section{ACKNOWLEDGEMENT}

I acknowledgment the support and help provided by my guide, parents and my friend.

\section{ABBREVIATIONS}

CP-Cerebral palsy; IUGR- intrauterine growth restriction.

\section{REFERENCES}

1. SCPE. Dev Med Child Neurol.2000;42:816-24.
2. Mutch L, Alberman E, Hagberg B, Kodama K, Perat MV. Cerebral palsy epidemiology: where are we now and where are we going? Dev Med Child Neurol. 1992;34(6):547-51.

3. Morris C. Definition and classification of cerebral palsy: A historical perspective. Dev Med Child Neurol Suppl. [Historical Article]. 2007;49(s109):3-7.

4. Himmelmann, Hagberg G. The changing panorama of cerebral palsy in Sweden. X. Prevalence and origin in the birth-year period 1995-1998. Acta Paediatrica.2010;99(9):1337-43.

5. AshwalS, Russman B, Practice Parameter: Diagnostic assessment of the child with cerebral palsy. Neurology. 2004;62(6):851-63.

6. Stanley F, Blair E, Alberman E. Cerebral palsies: Epidemiology and causal pathways, Vol 151. London (United Kingdom): MacKeith Press. 2000.

7. Murphy DJ, Johnson AM, Sellers S, MacKenzie IZ. Case-control study of antenatal and intrapartum risk factors for cerebral palsy in very preterm singleton babies. Lancet. 1995;346(8988):1449-54.

8. Oskoui M, Coutinho F, Dykeman J, Jette E, Pringsheim T. An update on the prevalence of cerebral palsy: A systematic review and meta-analysis. Dev Med Child Neurol. 2013;55(6):509-19.

9. Garne E, Dolk H, Krageloh-Mann I, Holst Ravn S, Cans C. Cerebral palsy and congenital malformations. Eur J Paediatr Neurol. 2008;12(2):82-8.

10. Blair E, Asedy F, Badawi N, Bower C. Is cerebral palsy associated with other defects other than cerebral defects? Dev Med Child Neurol. 2007;49(4):252-8.

11. McIntyre S, Blair E, Badawi N, Keogh J, Nelson K. Antecedents of cerebral palsy and perinatal death in term and late preterm singletons. Obstet Gynecol. 2013;122(4):869-77.

12. Grether JK, Nelson KB. Maternal infection and cerebral palsy in infants of normal birth weight. JAMA. 1997;278(3):207-11.

13. O'Callaghan ME, MacLennan AH, Gibson CS, et al. Epidemiologic associations with cerebral palsy. Obstet Gynecol. 2011;118(3):576-82.

14. WuYW,EscobarGJ,GretherJK,CroenLA, Greene JD, Newman TB. Chorioamnionitis and cerebral palsy in term and near-term infants. JAMA. 2003;290(20):2677-84.

15. BlairEM,NelsonKB.Fetalgrowthrestriction and risk of cerebral palsy in singletons born at least 35 weeks gestation. Am J Obstet Gynecol. 2015;212(4):520-e1.

16. Davies MJ, Moore VM, Willson KJ, et al. Reproductive technologies and the risk of birth defects. N Engl J Med. 2012;366(19):1803-13.

17. McMichael G, MacLennan AH, Gibson G, et al. Cytomegalovirus and EpsteinBarr virus may be associated with some cases of cerebral palsy. J Matern Fetal Neonatal Med. 2012;25(10):2078-81.

18. Polivka BJ, Nickel JT, Wilkins JR. Urinary tract infection during pregnancy: A riskfactor for cerebral palsy?JObstet Gynecol Neonatal Nurs. 1997;26(4):405-13.

19. Gibson CS, MacLennan AH, Hague WM, et al. For the South Australian Cerebral Palsy Research Group. Associations between inherited thrombophilias, gestational age, and cerebral palsy. Am J Obstet Gynecol. 2005;193(4):1437-43.

20. GibsonCS,MacLennanAH,GoldwaterPN, et al. The association between inheritedcytokine polymorphisms and cerebral palsy. Obstet Gynecol. 2006;194(3):674-e1.

21. Taylor F, National Institute of Neurological Disorders and Stroke (US). Office of Science and Health Reports. Cerebral palsy: hope through research. Bethesda, Md. The Institute. 2001. Accessed online September 28, 2005, at: http://www. ninds.nih.gov/disorders/cerebral_palsy/detail_cerebral_palsy.htm.

22. Neurologic disorders. In: The Merck manual of diagnosis and therapy. Accessed online September 8. 2005. http://www.merck.com/mrkshared/ mmanual/section19/chapter271/271b.jsp.

23. Taylor F. National Institute of Neurological Disorders and Stroke (U.S.) Office of Science and Health Reports. Cerebral palsy: Hope through research. Bethesda, Md. The Institute.2001. Accessed online September 28, 2005, at: http://www.ninds.nih.gov/disorders/cerebral_palsy/detail_cerebral_palsy.htm

24. Tilton $\mathrm{AH}$. Management of spasticity in children with cerebral palsy. Semin Pediatr Neurol.2004;11(1):58- 65.

25. Chung CY, Chen CL, Wong AM. Pharmacotherapy of spasticity in children with cerebral palsy. J FormosMed Assoc.2011;110(4):215-22.

26. Roche N, Even-Schneider A, Bussel B, et al. Management of increase in spasticity in patients with intrathecal baclofen pumps. Ann Readapt MedPhys.2007;50(2):93-9.

27. Mess SA, Kim S, Davison S, et al. Implantable baclofen pump as an adjuvant in treatment of pressure sores. Ann Plast Surg.2003;51(5):465-7.

28. Amirsalari S, Dalvand H, Dehghan L, et al. The efficacy of botulinum toxin type $A$ injection in the hamstring and calf muscles with and without serial foot

Indian Journal of Pharmacy Practice, Vol 11, Issue 2, Apr-Jun, 2018 
casting in gait improvement in children with cerebral palsy. Tehran Uni Med J.2011;69(8):509-17.

29. Dalvand H, Dehghan L, Feizy A, et al. The effect of foot serial casting along with botulinum toxin type-an injection on spasticity in children with cerebral palsy. J Kerman Uni Med Sci.2012;19(6):562-73. [in Persian]

30. Kinnett D. Botulinum toxin and injections in children: Technique and dosing issues. Am J Phys Med Rehabil. 2004;83(10):S59-64.
31. Jefferson RJ. Botulinum toxin in the management of cerebral palsy. Dev Med Child Neurol.2004;46(7):491-9

32. Yang TF, Fu CP, Kao NT, et al. Effect of botulinum toxin type an on cerebral palsy with upper limb spasticity. Am J Phys Med Rehabil.2003;82(4):284-9

33. Cerebral Palsy-An information guide for parents. CP booklet ISBN0958741654. 